\title{
THE SPECTRA OF UNBOUNDED HYPONORMAL OPERATORS
}

\author{
C. R. PUTNAM ${ }^{1}$
}

ABstract. A bounded operator $T$ on a Hilbert space is said to be completely hyponormal if $T^{*} T-T T^{*} \geqq 0$ and if $T$ has no nontrivial reducing space on which it is normal. If 0 is in the spectrum of such an operator $T$ and if the spectrum of $T$ near 0 is not "too dense," then the unbounded operator $T^{-1}$ acts as though it were bounded. In particular, under certain conditions, $T^{-1}$ has a rectangular representation with absolutely continuous real and imaginary parts whose spectra are the closures of the projections of the spectrum of $T^{-1}$ onto the coordinate axes.

1. A bounded operator $T$ on a Hilbert space $\mathfrak{H}$ is said to be hyponormal if

$$
T^{*} T-T T^{*}=D \geqq 0 .
$$

If $T$ has the Cartesian representation

$$
T=H+i J
$$

then condition (1.1) becomes

$$
H J-J H=-i C, \quad D=2 C \geqq 0 .
$$

It is known that the spectra of $H$ and $J$ are the (real) projections onto the real and imaginary axes of $\operatorname{sp}(T)$ (Putnam [2], also [3, p. 46]). The operator $T$ is said to be completely hyponormal if there is no nontrivial subspace of $\mathfrak{S}$ which reduces $T$ and on which $T$ is normal. In this case, both $H$ and $J$ are absolutely continuous (see [3, p. 42]).

If $T$ is hyponormal and if 0 is not in its spectrum, then the (bounded) operator $T^{-1}$ is also hyponormal (Stampfli [7]). If 0 belongs to the continuous spectrum of $T$ then $T^{-1}$ is unbounded, closed and has a dense range (Stone [9, pp. 40, 129]); further (Stampfli [8]),

$$
\mathfrak{D}_{T^{-1}} \subset \mathfrak{D}_{T^{-1}} \text { and }\left\|T^{-1 *} x\right\| \leqq\left\|T^{-1} x\right\| \quad \text { for } x \in \mathfrak{D}_{T^{-1}}
$$

Received by the editors March 8, 1971.

AMS 1970 subject classifications. Primary 47B20, 47B47; Secondary 47A10.

Key words and phrases. Hyponormal operators, absolutely continuous operators.

${ }^{1}$ This work was supported by a National Science Foundation research grant. 
The following result was proved in Putnam [5]:

THEOREM 1. Let 0 belong to the spectrum of the (bounded) completely hyponormal operator $T$ and suppose that for some number $a>0$ the two open disks $|z \pm i a|<$ a contain no points of $\operatorname{sp}(T)$. Then 0 is in the continuous spectrum of $T$ and $T^{-1}$ has a representation

$$
T^{-1}=K+i L, \quad K \text { and } L \text { selfadjoint, } L \text { bounded } .
$$

Hence $T^{-1 *}=K-i L$ and $($ by $(1.4))$,

$$
\left\|T^{-1 *} x\right\| \leqq\left\|T^{-1} x\right\| \text { for } x \in \mathfrak{D}_{T^{-1}}\left(=\mathfrak{D}_{T^{-1}}=\mathfrak{D}_{K}\right) .
$$

Also,

$K$ is absolutely continuous,

but, in general, $L$ need not be absolutely continuous.

In the present paper, it will be shown that if $T$ satisfies the conditions of Theorem 1 and if $\operatorname{sp}(T)$ is not too dense near the origin, then $T^{-1}$ behaves almost exactly like a bounded hyponormal operator. In fact, there will be proved the following two theorems:

THEOREM 2. Let T satisfy the hypothesis of Theorem 1. Then

$$
\mathrm{sp}(K)=\text { projection of } \mathrm{sp}\left(T^{-1}\right) \text { onto the real axis, }
$$

and

$$
\begin{aligned}
& \operatorname{sp}(L) \subset \text { closure of the (real) projection of } \operatorname{sp}\left(T^{-1}\right) \\
& \text { onto the imaginary axis. }
\end{aligned}
$$

If, in addition,

$$
\operatorname{sp}(K) \neq(-\infty, \infty)
$$

then

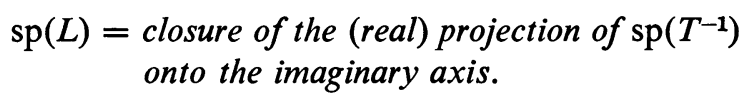

THEOREM 3. Let $T$ satisfy the hypothesis of Theorem 1 and, in addition, the condition

$$
\operatorname{meas}_{2}\left(\operatorname{sp}\left(T^{-1}\right)\right)<\infty
$$

Then $L\left(\mathfrak{D}_{K}\right) \subset \mathfrak{D}_{K}$ and

(1.13) $(K L-L K) x=-i M x, \quad x \in \mathfrak{D}_{K}, M$ bounded and $M \geqq 0$.

If, in addition, (1.10) is assumed, then

$L$ (as well as $K$ ) is absolutely continuous. 
It may be noted that since $T^{-1}$ in the above theorems is a closed operator, the set $\operatorname{sp}\left(T^{-1}\right)$ is closed (but unbounded) (see [9, p. 141]).

For $z \neq 0, z \in \operatorname{sp}(T)$ if and only if $z^{-1} \in \operatorname{sp}\left(T^{-1}\right)$ (cf. [5]). Also, the mapping $w=1 / z$ maps the circles $|z-b|=b$ ( $b$ real) into lines parallel to the imaginary axis. In view of (1.8), the condition (1.10) amounts then to supposing that there exists some real number $b \neq 0$ such that the circle $|z-b|=b$ intersects $\operatorname{sp}(T)$ in the single point $z=0$.

In order to illustrate the meaning of (1.12), suppose, for instance, that $T$ is hyponormal and that $\operatorname{sp}(T)$ lies between the curves $y= \pm a|x|^{b}$ where $a>0$ and $b>3$. Then $T$ clearly satisfies the hypothesis of Theorem 1 . In addition, the relation (1.12) holds. To see this, note that if $w=u+i v=$ $1 / z=1 /(x+i y)$ then $u=x /\left(x^{2}+y^{2}\right)$ and $v=-y /\left(x^{2}+y^{2}\right)$. Thus, for $x$ near 0 , $u \sim 1 \mid x$ and $|v| \sim a|x|^{-b-2}$, so that $\left.|v| \sim a|| u\right|^{b-2}$. Hence, near $z=\infty, \operatorname{sp}\left(T^{-1}\right)$ lies between the curves $v= \pm$ const $/|u|^{b-2}$ and so (1.12) holds.

An example given in [5] shows that $T$ in Theorem 2 may fail to satisfy (1.11) if (1.10) does not hold.

2. Before beginning the proofs of Theorems 2 and 3 , it will be convenient to give two lemmas.

LEMMA 1. Let $T$ be any bounded hyponormal operator given by (1.2) and (1.3) and let $H$ have the spectral resolution $H=\int \lambda d E_{\lambda}$. Let $\Delta$ be an open interval, $\alpha$ be any Borel set of the line, and let $r$ denote the distance between the sets $\Delta$ and $\alpha$. Then

$$
\|E(\alpha) J E(\Delta)\| \leqq|\Delta|^{1 / 2}\|J\| / r^{1 / 2} .
$$

Proof. See Putnam [6].

Next, assume that $T$ satisfies the hypothesis of Theorem 1, so that (1.5) holds, and let $K$ have the spectral resolution

$$
K=\int \lambda d G_{\lambda} .
$$

If $\Delta=(a, b)$, where $-\infty<a<b<\infty$, then the operator

$$
G(\Delta) T^{-1} G(\Delta)=\int_{\Delta} \lambda d G_{\lambda}+i G(\Delta) L G(\Delta)
$$

is bounded on the Hilbert space $G(\Delta) \mathfrak{H}$. It follows from (1.6) that

$$
\left\|T^{-1} x\right\|^{2}-\left\|T^{-1 *} x\right\|^{2}=2 i[(L x, K x)-(K x, L x)] \geqq 0, \quad x \in \mathfrak{D}_{K} .
$$

Since $G(\Delta) \mathfrak{H} \subset \mathfrak{D}_{K}$, it is clear that $G(\Delta) T^{-1} G(\Delta)$ is hyponormal on $G(\Delta) \mathfrak{H}$. It follows from Putnam [4] that the closed set $\operatorname{sp}\left(G(\Delta) T^{-1} G(\Delta)\right)$ is a 
monotone nondecreasing function of $\Delta$ in the sense that

$$
\operatorname{sp}\left(G\left(\Delta_{1}\right) T^{-1} G\left(\Delta_{1}\right)\right) \subset \operatorname{sp}\left(G\left(\Delta_{2}\right) T^{-1} G\left(\Delta_{2}\right)\right) \text { when } \Delta_{1} \subset \Delta_{2} .
$$

LEMMA 2. If T satisfies the hypothesis of Theorem 1 , then

$$
\operatorname{sp}\left(T^{-1}\right)=\Omega,
$$

where $\Omega=$ closure $\left\{\bigcup_{\Delta} \operatorname{sp}\left(G(\Delta) T^{-1} G(\Delta)\right)\right\}, \Delta$ being any finite open interval.

Proof. First, it will be shown that

$$
\Omega \subset \operatorname{sp}\left(T^{-1}\right) .
$$

Let $z=s+i t \in \operatorname{sp}\left(G(\Delta) T^{-1} G(\Delta)\right)$ for some finite open interval $\Delta$. Since $\mathrm{sp}\left(T^{-1}\right)$ is closed, the inclusion (2.7) will be established if it is shown that $z \in \operatorname{sp}\left(T^{-1}\right)$. In view of $(2.5), z \in \operatorname{sp}\left(S_{n}\right)$, for sufficiently large positive integers $n$, where $S_{n}=G_{n} T^{-1} G_{n}$ and $G_{n}=G((-n, n))$. Hence there exist unit vectors $x_{n}=G_{n} x_{n}$ for which

$$
\left(S_{n}-z I\right)^{*} x_{n}=G_{n}\left(T^{-1}-z I\right)^{*} G_{n} x_{n} \rightarrow 0, \text { as } n \rightarrow \infty .
$$

It is clear that $x_{n} \in \mathfrak{D}_{K}\left(=\mathfrak{D}_{T^{-1 *}}=\mathfrak{D}_{T^{-1}}\right)$ and that

$$
\left(T^{-1}-z I\right)^{*} x_{n}=\left(S_{n}-z I\right)^{*} x_{n}-i\left(I-G_{n}\right)(L-t I) G_{n} x_{n} .
$$

It will be shown that $z \in \operatorname{sp}\left(T^{-1}\right)$ if it is shown that $\left(T^{-1}-z I\right)^{*} x_{n} \rightarrow 0$, that is, in view of (2.8), if it is shown that

$$
\left(I-G_{n}\right)(L-t I) G_{n} x_{n} \rightarrow 0, \text { as } n \rightarrow \infty .
$$

By (2.8) and (1.5),

$$
\left\|(K-s I) x_{n}\right\|^{2}=\int_{-n}^{n}(\lambda-s)^{2} d\left\|E_{\lambda} x_{n}\right\|^{2} \leqq \text { const } \quad(<\infty) .
$$

Consequently, for every $\varepsilon>0$, there exists a positive integer $N=N_{\varepsilon}$ such that $\left\|\left(G_{n}-G_{N}\right) x_{n}\right\|<\varepsilon$ for $n>N$. Hence,

$$
\begin{aligned}
& \left\|\left(I-G_{n}\right)(L-t I) G_{n} x_{n}\right\| \\
& \quad \leqq \varepsilon\|(L-t I)\|+\left\|\left(I-G_{n}\right)(L-t I) G_{N}\right\|, \quad n>N=N_{\varepsilon} .
\end{aligned}
$$

Let $\Delta$ denote any finite open interval and let $\alpha$ be a bounded Borel set of the line. Suppose that $\Delta$ and $\alpha$ are at a distance $r$ apart and suppose that $\delta$ is a finite open interval containing both $\Delta$ and $\alpha$. Then $G(\delta) T^{-1} G(\delta)$ of (2.3) is bounded and hyponormal, and, by (2.1),

$$
\|G(\alpha) G(\delta)(L-t I) G(\delta) G(\Delta)\| \leqq|\Delta|^{1 / 2}\|G(\delta)(L-T I) G(\delta)\| / r^{1 / 2} .
$$


If now $\delta=\delta_{k}=(-k, k)$ and $k \rightarrow \infty$, one obtains

$$
\|G(\alpha)(L-t I) G(\Delta)\| \leqq|\Delta|^{1 / 2}\|(L-t I)\| / r^{1 / 2}, \quad \operatorname{dist}(\alpha, \Delta)=r,
$$

of any bounded Borel set $\alpha$. Since the right side of this inequality is independent of $\alpha$, it is clear that (2.13) holds without the boundedness restriction on $\alpha$. Consequently, $\left\|\left(I-G_{n}\right)(L-t I) G(\Delta)\right\| \rightarrow 0$, as $n \rightarrow \infty$, for any fixed finite interval $\Delta$. Relation (2.10) now follows from (2.12), and so (2.7) is proved.

In order to complete the proof of Lemma 2 , it remains to be shown that $\operatorname{sp}\left(T^{-1}\right) \subset \Omega$. To this end, suppose that $z \notin \Omega$; it will be proved that $z \notin \operatorname{sp}\left(T^{-1}\right)$. Since $\Omega$ is closed, then $d=\operatorname{dist}(z, \Omega)>0$. Further, if $\Delta$ is any finite open interval then $G(\Delta) T^{-1} G(\Delta)$ is a bounded hyponormal operator on $G(\Delta) \mathfrak{H}$, and so $\left\|G(\Delta)\left(T^{-1}-z I\right)^{*} G(\Delta) x\right\| \geqq d\|G(\Delta) x\|$ for any $x \in \mathfrak{H}$. On putting $\Delta=(-k, k)$ and letting $k \rightarrow \infty$, one obtains, for $x \in \mathfrak{D}_{T^{-1}}\left(=\mathfrak{D}_{T^{-1 *}}=\mathfrak{D}_{K}\right)$, $\left\|\left(T^{-1}-z I\right) x\right\| \geqq\left\|\left(T^{-1}-z I\right)^{*} x\right\| \geqq d\|x\|$, so that $z \notin \operatorname{sp}\left(T^{-1}\right)$. The proof of Lemma 2 is now complete.

3. Proof of Theorem 2. Relation (1.8) can be deduced from a result of Clancey [1, p. 47]. It can also be concluded from (2.6) and the fact that the spectrum of $K G(\Delta)=\int_{\Delta} \lambda d G_{\lambda}$ is the projection of the spectrum of $G(\Delta) T^{-1} G(\Delta)$, as an operator on $G(\Delta) \mathfrak{H}$, onto the real axis.

In order to prove (1.9), let $t \in \operatorname{sp}(L)$. If $G_{n}=G((-n, n))$, then $G_{n} L G_{n} \rightarrow L$ (strongly) as $n \rightarrow \infty$. Since $L$ is selfadjoint, there exist real numbers $t_{n} \in \operatorname{sp}\left(G_{n} L G_{n}\right)$ satisfying $t_{n} \rightarrow t$ as $n \rightarrow \infty$. As a consequence of the projection properties of the spectrum of a hyponormal operator (cf. [3, p. 46]) there exist real numbers $s_{n}$ such that $s_{n}+i t_{n} \in \operatorname{sp}\left(G_{n} T^{-1} G_{n}\right)$. It follows from (2.6) that $s_{n}+i t_{n} \in \operatorname{sp}\left(T^{-1}\right)$ for all $n$, and (1.9) now follows.

That relation (1.10) implies the reverse inclusion of (1.9) follows from Clancey [1, p. 45]. This result together with (1.9) then yields (1.11) and the proof of Theorem 2 is complete.

4. Proof of Theorem 3. Let $\Delta$ be a bounded open interval, so that $G(\Delta) T^{-1} G(\Delta)$ of $(2.3)$ is a bounded hyponormal operator on $G(\Delta) \mathfrak{G}$. In view of (2.4) and the fact that $G(\Delta) \mathfrak{H} \subset \mathfrak{D}_{K}$,

$$
i\left(K_{\Delta} L_{\Delta}-L_{\Delta} K_{\Delta}\right)=M^{(\Delta)} \geqq 0,
$$

where $K_{\Delta}=G(\Delta) K G(\Delta)(=K G(\Delta)), L_{\Delta}=G(\Delta) L G(\Delta)$ and

$$
M^{(\Delta)}=G(\Delta) M^{(\Delta)} G(\Delta) .
$$

It follows from [4] that

$$
\pi\left\|M^{(\Delta)}\right\| \leqq \operatorname{meas}_{2} \operatorname{sp}\left(G(\Delta) T^{-1} G(\Delta)\right) .
$$


Consequently, from (2.6) and the assumption (1.12) of Theorem 3,

$$
\pi\left\|M^{(\Delta)}\right\| \leqq \operatorname{meas}_{2}\left(\operatorname{sp}\left(T^{-1}\right)\right)<\infty .
$$

But for any $x \in \mathfrak{D}_{K}, \quad i\left[\left(L_{\Delta} x, K_{\Delta} x\right)-\left(K_{\Delta} x, L_{\Delta} x\right)\right]=\left(M^{(\Delta)} x, x\right)$. Since $G(\Delta) \rightarrow I$ (strongly) as $\Delta \rightarrow(-\infty, \infty)$, it is clear that $\lim \left(M^{(\Delta)} x, x\right)$, as $\Delta \rightarrow(-\infty, \infty)$, exists for all $x \in \mathfrak{D}_{K}$. Since, by $(4.3),\left\|M^{(\Delta)}\right\| \leqq$ const (independent of $\Delta$ ), and since $\mathfrak{D}_{K}$ is dense in $\mathfrak{H}$, it follows that $\lim \left(M^{(\Delta)} x, x\right)$, as $\Delta \rightarrow(-\infty, \infty)$, exists for all $x$ in $\mathfrak{H}$. Further, since the operators $M^{(\Delta)}$ are selfadjoint, one sees that $\lim \left(M^{(\Delta)} x, y\right)$, as $\Delta \rightarrow(-\infty, \infty)$, exists for $x, y$ in $\mathfrak{H}$. Thus, $M=\mathrm{w}-\lim M^{(\Delta)}$ (weak limit), as $\Delta \rightarrow(-\infty, \infty)$, that is,

$$
i\left(K_{\Delta} L_{\Delta}-L_{\Delta} K_{\Delta}\right)=M^{(\Delta)} \stackrel{\mathrm{w}}{\rightarrow} M
$$

$$
\text { ( } M \text { bounded, } M \geqq 0), \Delta \rightarrow(\infty, \infty) .
$$

Let $\delta$ be any open interval containing a fixed open interval $\Delta$. It follows from (4.1) that $G(\Delta) M^{(\delta)} G(\Delta)=M^{(\Delta)}$ and hence, on letting $\delta \rightarrow(-\infty, \infty)$, $M^{(\Delta)}=\mathrm{w}-\lim G(\Delta) M^{(\delta)} G(\Delta)$; thus, by $(4.4), M^{(\Delta)}=G(\Delta) M G(\Delta)$. As $\Delta \rightarrow(-\infty, \infty), G(\Delta)$ converges strongly to $I$, and hence $M^{(\Delta)}$ converges strongly to $M$. Thus, by (4.4),

$$
i\left(K_{\Delta} L_{\Delta}-L_{\Delta} K_{\Delta}\right)=M^{(\Delta)} \stackrel{\mathrm{s}}{\rightarrow} M, \quad \Delta \rightarrow(-\infty, \infty) .
$$

Further, if $x \in \mathfrak{D}_{K}$, it is clear that $K_{\Delta} x \rightarrow K x$ and $L_{\Delta} K_{\Delta} x \rightarrow L K x$ as $\Delta \rightarrow$ $(-\infty, \infty)$. Consequently, it follows from (4.5) that the vectors $K_{\Delta} L_{\Delta} x=$ $K\left(L_{\Delta} x\right)$ converge strongly as $\Delta \rightarrow(-\infty, \infty)$. But $L_{\Delta} x \rightarrow L x$ and so, since $K$ is selfadjoint (hence closed), $L x \in \mathfrak{D}_{K}$ and $K\left(L_{\Delta} x\right) \rightarrow K L x$. This proves (1.13).

There remains then to prove (1.14) under the assumption (1.10). But it follows from (1.13) and an application of a result of [3, p. 39], that the subspace, $\mathfrak{H}_{a}(L)$, of $\mathfrak{S}$ on which $L$ is absolutely continuous (cf. [3, p. 19]) contains the least subspace of $\mathfrak{S}$ reducing $K$ and $L$ (that is, reducing $T^{-1}$ ) and containing the range of $M$. Thus, if $\mathfrak{H}_{a}(L) \neq \mathfrak{H}$, then (cf. (2.4) and (1.13)) $T^{-1}$, hence also $T$, would be normal on $H_{a}(L)^{\perp}$. Since $T$ is completely hyponormal, this yields a contradiction.

\section{REFERENCES}

1. K. F. Clancey, Spectral properties of semi-normal operators, Thesis, Purdue University, Lafayette, Ind., 1969.

2. C. R. Putnam, On the spectra of semi-normal operators, Trans. Amer. Math. Soc. 119 (1965), 509-523. MR 32 \#2913.

3. - Commutation properties of Hilbert space operators and related topics, Ergebnisse der Mathematik und ihrer Grenzgebiete, Band 36, Springer-Verlag, New York, 1967. MR 36 \#707.

4. - An inequality for the area of hyponormal operators, Math. Z. 116 (1970), 323-330. 
5. C. R. Putnam, Unbounded inverses of hyponormal operators, Pacific J. Math. 35 (1970), 755-762.

6. - A similarity between hyponormal and normal spectra, Illinois J. Math. (to appear).

7. J. G. Stampfli, Hyponormal operators and spectral density, Trans. Amer. Math. Soc. 117 (1965), 469-476. MR 30 \#3375.

8. — Analytic extensions and spectral localization, J. Math. Mech. 16 (1966), 287-296. MR 33 \#4687.

9. M. H. Stone, Linear transformations in Hilbert space and their applications to analysis, Amer. Math. Soc. Colloq. Publ., vol. 15, Amer. Math. Soc., Providence, R.I., 1932.

Department of Mathematics, Purdue University, LafayetTe, Indiana 47907 